\title{
Identification of androgenic gland microRNA and their target genes to discover sex-related microRNA in the oriental river prawn, Macrobrachium nipponense
}

\author{
S.B. Jin ${ }^{1,2}$, H.T. Fu' ${ }^{1,2}$, S.F. Jiang ${ }^{2}$, Y.W. Xiong ${ }^{2}$, H. Qiao' ${ }^{2}$, W.Y. Zhang ${ }^{2}$, Y.S. Gong ${ }^{2}$ \\ and $\mathrm{Y} . \mathrm{Wu}^{2}$ \\ ${ }^{1}$ Wuxi Fisheries College, Nanjing Agricultural University, Wuxi, China \\ ${ }^{2}$ Key Laboratory of Freshwater Fisheries and Germplasm Resources Utilization, \\ Ministry of Agriculture, Freshwater Fisheries Research Center, \\ Chinese Academy of Fishery Sciences, Wuxi, China \\ Corresponding author: H.T. Fu \\ E-mail: fuht@ffrc.cn
}

Genet. Mol. Res. 14 (4): 18396-18406 (2015)

Received August 16, 2015

Accepted October 30, 2015

Published December 23, 2015

DOI http://dx.doi.org/10.4238/2015.December.23.27

\begin{abstract}
The oriental river prawn, Macrobrachium nipponense, is an important aquaculture species in China. The androgenic gland produces hormones that play crucial roles in the differentiation of crustaceans to the male sex. MicroRNA (miRNA) post-transcriptionally regulates many protein-coding genes, influencing important biological and metabolic processes. However, currently, there is no published data identifying miRNA in M. nipponense. In this study, we identified novel miRNA in the androgenic gland of $M$. nipponense. Using the high-throughput Illumina Solexa system, 1077 miRNA were identified from small RNA libraries by aligning with the de novo androgenic gland transcriptome of $M$. nipponense (obtained from RNA-Seq) and the sequences in the miRBase21 database. A total of 8,248, 76,011, and 78,307 target genes were predicted in the EST and SRA sequences provided in the NCBI database, and the androgenic gland transcriptome of $M$. nipponense, respectively. Some potential sex-
\end{abstract}


related miRNA were identified based on the function of the predicted target genes. The results of our study provide new information regarding the miRNA expression in $M$. nipponense, which could be the basis for further genetic studies on decapod crustaceans.

Key words: Macrobrachium nipponense; Androgenic gland; microRNA; Targeted genes; Sex-determination

\section{INTRODUCTION}

The oriental river prawn, Macrobrachium nipponense (Crustacea; Decapoda; Palaemonidae), is an important commercial prawn species that is widely distributed in freshwater and low-salinity estuarine regions in China and other Asian countries (Cai and Shokita, 2006; Feng et al., 2008; Ma et al., 2011). It is an important fishery resource in China, with an annual aquaculture production of 205,010 tons (Bureau of Fishery, Ministry of Agriculture, 2009). As with many other Macrobrachium species, the male counterparts grow faster and gain more weight during the time of harvest than female river prawns. Thus, culturing of all-male populations would be beneficial for economic purposes.

The androgenic gland is found in most crustacean species. It produces hormones that play crucial roles in the development of the testes and male sexual characteristics (Sagi et al., 1990). In Macrobrachium rosenbergii, males undergo sex reversal to females after their androgenic gland is ablated. An all-male population was generated by mating "reversal females" with normal male $M$. rosenbergii (Sagi et al., 1986, 1990; Sagi and Cohen, 1990). As the ablation or implantation of the androgenic gland during a certain stage of development can result in sex reversal to male or female (Sagi and Cohen, 1990; Malecha et al., 1992; Manor et al., 2004), the expression pattern of androgenic gland hormone genes in crustaceans have received much attention over the past few years (Tomer et al., 2009, 2011; Tomer and Sagi, 2012; Ma et al., 2013). Studies into the androgenic gland of $M$. nipponense have also been popular in the recent years. The androgenic gland transcriptome of $M$. nipponense has been previously constructed (Jin et al., 2013), and used to analyze many sex-related homologous genes and novel genes, which helped outline the basic sex-determination mechanism of M. nipponense (Zhang et al., 2013a,b,c; Jin et al., 2014; Li et al., 2015b).

MicroRNA (miRNA) are 21-22 nucleotide non-coding RNA that play an important role in the regulation of gene expression, by degrading target mRNA or repressing targeted gene translation in both animals and plants (Bartel, 2004). One miRNA may regulate the expression of several genes expression; alternately, the expression of a single gene requires several miRNA to work synchronously. Lin-4 and let-7 are the first miRNA to be identified in Caenorhabditis elegans using a genetic screening method (Lee et al., 1993; Wightman et al., 1993). An increasing number of reports have suggested the role of miRNA in diverse biological functions, including embryo formation, organogenesis, cell death, cell proliferation, lipid metabolism, and immune development (Wightman et al., 1993; Xu et al., 2003; Johnson et al., 2005). To date, however, the miRNA genes of $M$. nipponense have not been identified despite shrimp miRNA having been extensively characterized.

Macrobrachium producers face stiff market competition; therefore, there is an immediate need for improvements in river prawn genetic traits and production techniques, which could help obtain a greater profit. Therefore, the long-term goals of the $M$. nipponense aquaculture industry include making genetic improvements to, and gaining a better understanding of, the sexdifferentiation mechanism in this species. In this study, an androgenic gland miRNA transcriptome 
was constructed using high-throughput Illumina Solexa analysis, in order to regulate the expression of sex-related genes in $M$. nipponense. The results of this study could contribute to elucidating the role of miRNA in regulating the expression of sex-related genes, which could lead to the advancement of analysis of the sex-determination mechanism in M. nipponense.

\section{MATERIAL AND METHODS}

\section{Prawn and tissue preparation}

A total of 100 healthy adult male $M$. nipponense, with a wet weight ranging from 5.12 to $6.93 \mathrm{~g}$ (average $=6.24 \mathrm{~g}$ ), and a total length ranging from $6.27 \mathrm{~cm}$ to $7.56 \mathrm{~cm}$ (average $=6.97 \mathrm{~cm}$ ),

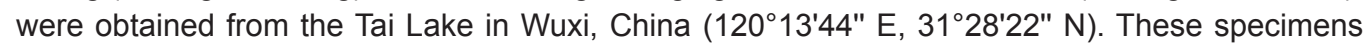
were transferred to a $500 \mathrm{~L}$ tank and maintained in aerated freshwater at room temperature $\left(26^{\circ} \mathrm{C}\right)$ with sufficient oxygen for $72 \mathrm{~h}$ prior to tissue collection.

\section{Sample collection and RNA extraction}

For miRNA sequencing, androgenic glands were collected from $100 \mathrm{M}$. nipponense in $1 \mathrm{X}$ phosphate buffered saline (PBS) under an Olympus SZX16 microscope (Olympus, Tokyo, Japan). The androgenic gland tissues were immediately frozen in liquid nitrogen, and stored at $-80^{\circ} \mathrm{C}$ until further use, in order to prevent total RNA degradation. Total RNA was extracted using a TRIzol Reagent Total RNA Isolation Kit (Invitrogen; Life Technologies, Carlsbad, CA, USA) according to the manufacturer protocols. The ratio of optical densities (OD) at 260 and $280 \mathrm{~nm}(\mathrm{OD} 260 / 280)$ and 260 and $230 \mathrm{~nm}$ (OD260/230) should range from 1.8 to 2.0 and $>2.0$, respectively, in order to ensure the purity of the RNA sample. In order to guarantee the quality of the transcriptome, the total volume of the RNA sample was maintained at $>5 \mu \mathrm{g}$. RNA integrity was confirmed using an Agilent Bioanalyzer (Agilent Technologies; Life Technologies) with a minimum RNA integrity number (RIN) of 8.0. The RNA of the androgenic glands of $100 \mathrm{M}$. nipponense were pooled to provide sufficient RNA for transcriptome sequencing.

\section{Small RNA library preparation and sequencing}

Illumina Hiseq2500 (Illumina Inc., San Diego, CA, USA) was used for in-depth sequencing of the small RNA libraries, using total RNA as the starting material. Small RNA were first isolated from the total RNA, and a pair of Illumina proprietary adaptors was ligated to their 5'- and 3'-ends using the Truseq ${ }^{\mathrm{TM}}$ Small RNA sample prep kit, according to the manufacturer instructions (Illumina Inc.). Adaptor-ligated small RNAs were then reverse transcribed to create cDNA constructs using the Truseq ${ }^{\mathrm{TM}}$ Small RNA sample prep kit (Illumina Inc.). The generated small cDNA libraries were amplified by 11-12 cycles of PCR and purified on a 6\% Novex TBE PAGE gel (1.0 mm, 10 well; Invitrogen). The PCR reaction conditions: the reactions are incubated at $94 \mathrm{C}$ for $1 \mathrm{~min}$, and then cycled 12 times at $94 \mathrm{C}$ for $45 \mathrm{~s}, 55 \mathrm{C}$ for $45 \mathrm{~s}$, and $72 \mathrm{C}$ for $45 \mathrm{~s}$. This is followed by 3 min incubation at $72 \mathrm{C}$. The library was sequenced using the purified PCR product (MajorBio, Shanghai, China).

\section{Data cleaning, length distribution, and sequence data analysis}

An initial filtering step was performed to remove low quality reads using SeqPrep (https:// 
github.com/jstjohn/SeqPrep) and Sickle (https://github.com/najoshi/sickle). Low quality reads include reads with 5' primer contaminants, reads without the 3 ' primer or the insert tag, reads with a poly-A tail or with $\mathrm{N}>10 \%$, and reads shorter than 18 nucleotide. The length distribution of the clean reads between 18 and 32 bp was then summarized. Identical reads from clean reads were combined in order to obtain unique reads for further small RNA analysis.

Non-coding RNA, such as rRNA, tRNA, snRNA, snoRNA, and scRNA were identified by aligning the obtained sequence to similar sequences in Rfam 11.0 (http://Rfam.sanger.ac.uk/) and the NCBI database (http://blast.ncbi.nlm.nih.gov/). The reads were classified into different categories based on the sequence similarity, and the remnant reads were also aligned using the Basic Local Alignment Search Tool (BLAST) against corresponding sequences in miRBase 21 (http://www.mirbase.org/) and the reference genome, in order to identify known miRNA.

\section{Target gene prediction}

Miranda (http://www.miRNA.org/miRNA/home.do) was used to predict the target, in accordance with the following criteria in seed matches: nucleotides 1-9 from the 5'-end and G-U content $<3$. The reference genomes, used in the prediction of target genes, included the EST and SRA sequences of $M$. nipponense obtained from NCBI, and the androgenic gland transcriptome of M. nipponense. Gene targets of miRNA were identified using an appropriate prediction software. All miRNA targets were categorized into functional classes using the gene ontology hierarchy (http:// www.geneontology.org and DAVID Bioinformatics Resources).

\section{RESULTS}

\section{Length distribution}

The total number of raw reads of the androgenic gland of $M$. nipponense small RNA library, obtained by high throughput Solexa sequencing using HighSeq 2500, was 25,864,140 $(22,549,954)$ high quality reads. The impurities in the raw reads, including 5 '-primer contaminants, lack of insert tags, low quality reads, reads with poly-A tail only, oversized insertion, and short reads, were discarded. The length distributions of the clean reads were then summarized. The total number of reads with a length of 18-32 nt was 17,574,958. Most reads were 22 and $21 \mathrm{nt}$ in length; the second highest number of reads were 20 and $23 \mathrm{nt}$ in length (Figure 1).

\section{Known miRNA abundance and sequences identification}

miRNA, rRNA, snoRNA, snRNA, scRNA, tRNA, repeat DNA, and unannotated RNA were annotated by comparing the small RNA sequences with the sequences in the GenBank and RFam 10.1 databases. Non-miRNA sequences, including tRNA, rRNA, scRNA, snRNA, and snoRNA sequences, were removed. miRNA sequences accounted for $22 \%$ of the total reads. rRNA, tRNA, and other RNA sequences accounted for 18,4 , and $1 \%$ of the total reads, respectively; the remaining $55 \%$ of the total reads were unmatched (Figure 2). The known miRNA in the androgenic gland of $M$. nipponense were identified by aligning the mappable sequences to known miRNA precursors and mature miRNA of Arthropoda species in the miRBase database. By allowing no more than two mismatches between sequences, 1077 mature miRNA were identified. The miRNA 
count in the androgenic gland of $M$. nipponense ranged from 1 to $1,766,725$. miR-100, bantam, miR-184, miR-315, let-7, miR-315, miR-8-5p, miR-10, miR-8-3p, and miR-12 were some of the most abundant miRNA observed, with a total number of reads greater than 60,000 .

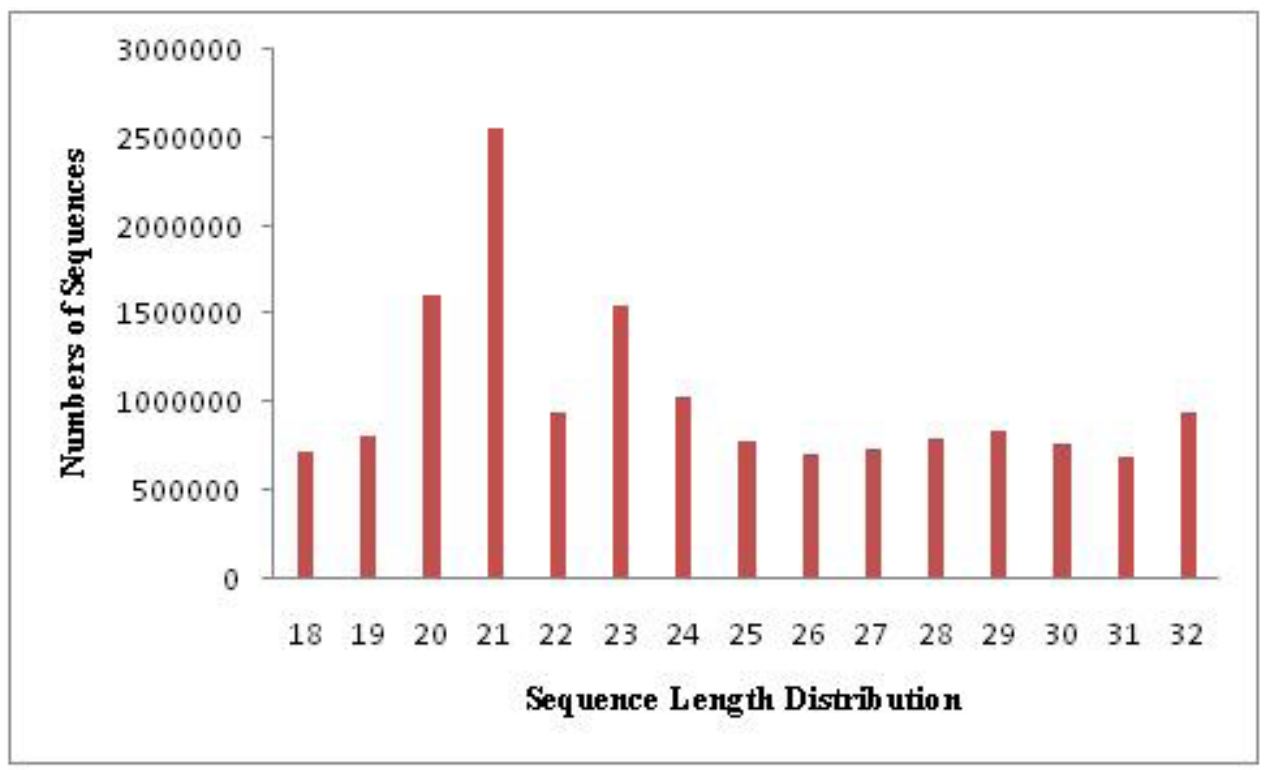

Figure 1. Size distribution of mapped reads in the androgenic gland of Macrobrachium nipponense.

\section{Pie Chart of AG}

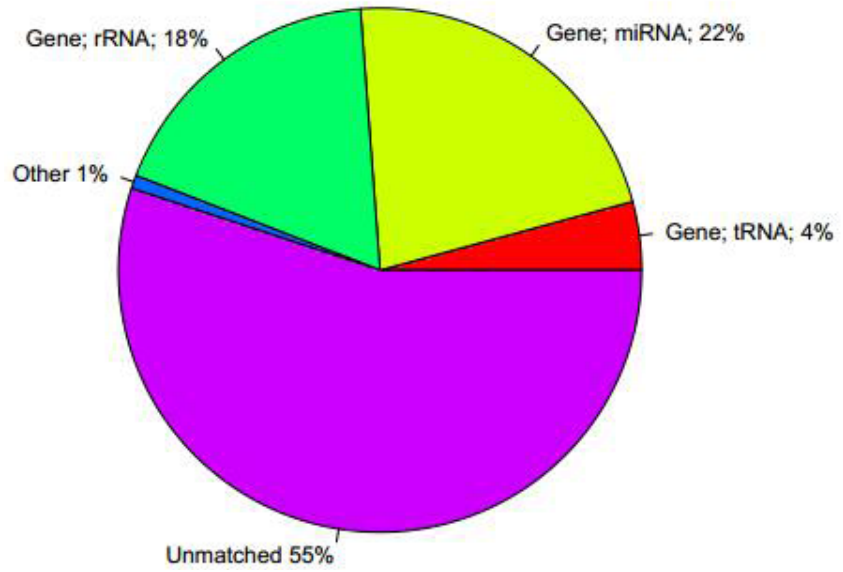

Figure 2. Pie chart of data filtering and database mapping in the androgenic gland of Macrobrachium nipponense. RFam: rRNA, tRNA, snRNA, snoRNA, and others. 


\section{Target gene prediction}

Because of the lack of whole genome data of $M$. nipponense, we have used the known sequences of $M$. nipponense as a reference to predict the target genes. A total of $8,248,76,011$, and 78,307 target genes were predicted in the EST and SRA sequences in NCBI, and in the androgenic gland transcriptome of $M$. nipponense, respectively. In order to fully elucidate the function of the novel target genes identified as being potentially regulated by miRNA, the target genes were enriched by Gene Ontology. The matched contigs were comprised of 60 functional groups. Those potential target genes were mainly involved in cellular process, binding, metabolic process, and catalytic activity, with a total number of contigs greater than 4,000 (Figure 3). Several sex-related genes of $M$. nipponense were identified, including the insulin-like androgenic gland, sex-lethal, and transformer-2 genes. Several sex-related miRNA were identified in M. nipponense using these sex-related genes as target genes (Table 1).

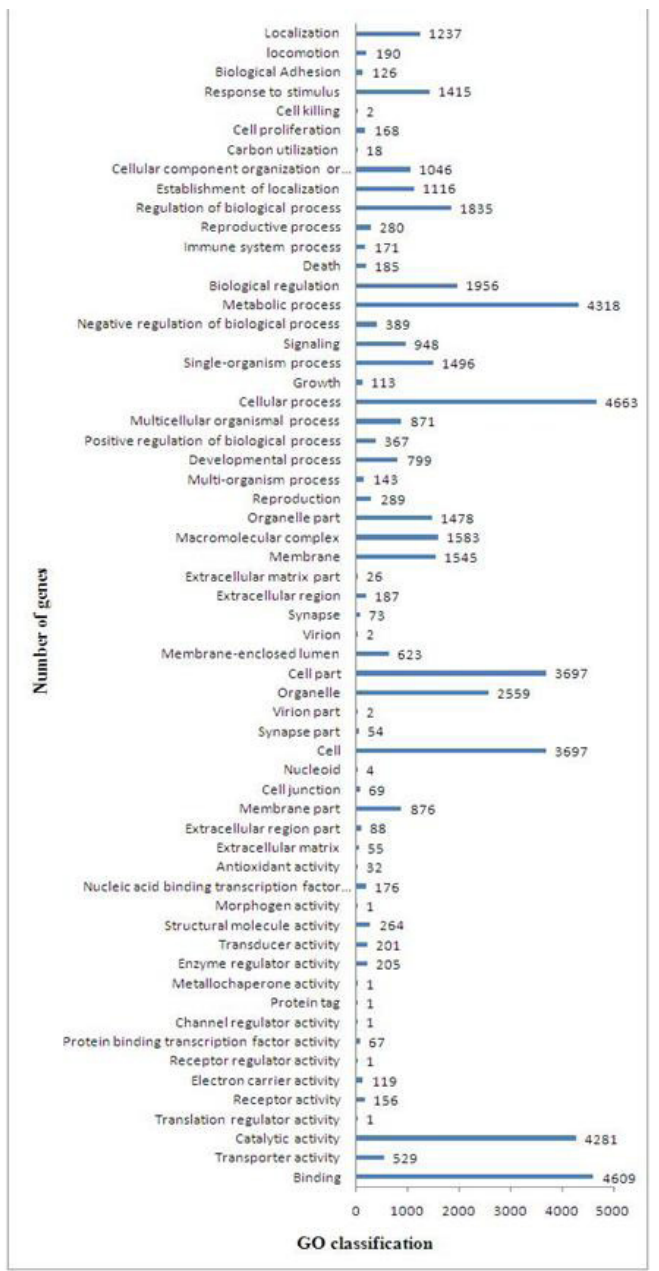

Figure 3. GO analysis of miRNA target genes in the androgenic gland of Macrobrachium nipponense. 


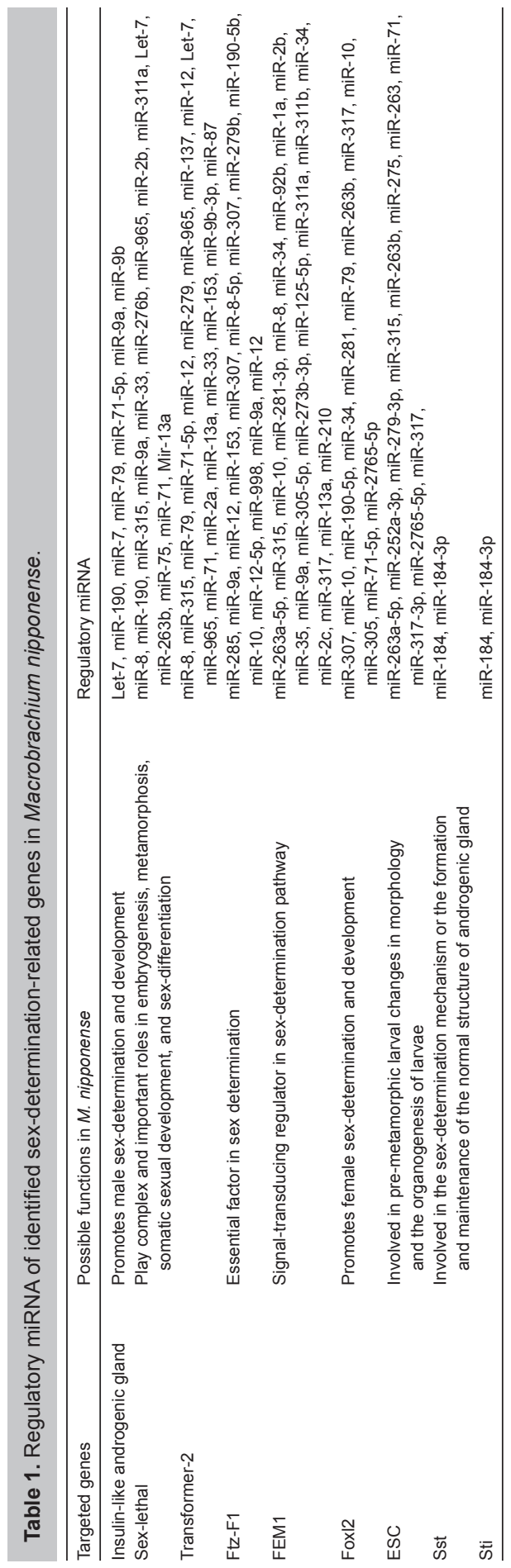




\section{DISCUSSION}

Over six million miRNA have now been identified in the androgenic gland of $M$. nipponense (miRBase v.20). To the best of our knowledge, there is no available information regarding the miRNA in $M$. nipponense. So far, only the miRNA transcriptome of a mixture of organisms have been reported in Macrobrachium rosenbergii (Tan et al., 2013) among the various Macrobrachium species. In this study, we attempted to identify small RNA from the androgenic gland of $M$. nipponense, as the first step towards understanding the regulatory roles of small RNA in the sexdetermination mechanism, because of the importance of the androgenic gland in sex-differentiation and development of males in the crustacean species.

Length distribution analysis plays an essential role in the in-depth analysis of the compositions of small RNA samples. Normally, the length of small RNA varies from 18-nt to 30-nt. miRNA, siRNA, and piRNA are normally 21-22-nt, 24-nt, and 30-nt long. The length distribution varies between plants and animals. The peak of plant small RNA is at a range of 21-24-nt, while that of animal small RNA is 22-nt. In this study, 20-23-nt small RNA were abundantly expressed in the androgenic gland of these crustaceans. The length of predicted miRNA in Penaeus monodon was reported to range from 16-nt to 23-nt (Pitipol et al., 2013), while a peak miRNA ranging from 21 to 23-nt, represented the typical miRNA of dicer-processed products in Eriocheir sinensis (Song et al., 2014). The peak small RNA size of 22-nt observed in the gill and hepatopancreas of Macrobrachium rosenbergii revealed that the miRNA is enriched in this small RNA library (Tan et al., 2013). Therefore, the 20-23-nt peak observed in this study indicates the identification of miRNA in the androgenic gland of $M$. nipponense, which may help advance research in the sexdetermination mechanism of $M$. nipponense.

A total of 1,077 mature miRNA were identified when the sequenced small RNA were aligned with known miRNA precursors and mature miRNA of Arthropoda species uploaded to the miRBase database. The miRNA count in the androgenic gland of $M$. nipponense ranged from 1 to $1,766,725$. These highly expressed miRNA are believed to be involved in the sex-determination mechanism in M. nipponense, as the androgenic gland plays a major role in sex differentiation and the development of males in the crustacean species.

A series of miRNA have been identified as sex-determination-related miRNA, containing important sex-determination-related genes of $M$. nipponense, through the prediction of target genes. In previous studies, the let-7 gene family in mammals have been reported to regulate the expression of major cytokine-inducible proteins in immune response against microbial infection (O'Hara et al., 2010; Asgari, 2011; Schulte et al., 2011; Asgari, 2012). In this study, Let-7 was predicted to be involved in the regulation of some important sex-determination-related genes of $M$. nipponense. The function of insulin-like androgenic gland (IAG) is believed to be similar to that of the isopod AG hormone, considered as a key regulator of male sex-determination and development (Tomer and Sagi, 2012). In M. nipponense, IAG was reported to be involved in sex-determination (Ma et al., 2013), and has been shown to regulate the expression of three crustacean genes belonging to the hyperglycemic hormone superfamily ( $\mathrm{Li}$ et al., 2015a) as well as the gene coding for insulin-like androgenic gland hormonebinding protein (Li et al., 2015b). Four genes, sex-lethal (SxI), transformer (Tra), transformer-2 (Tra-2), and double-sex $(D s x)$, regulate the sex-determination pathway in Drosophila melanogaster. SxI and Tra-2 have been reported to play complex and important roles in embryogenesis, metamorphosis, somatic sexual development, and sex-differentiation in M. nipponense (Zhang et al., 2013a,b). In this study, mir-2a, miR-33, miR-71-5p, miR-965, and miR-315 were also predicted to regulate the 
expression of $S x I$ and Tra-2, indicating a regulatory relationship between $S x I$ and Tra-2, which must be analyzed in further research.

We also predicted that $\mathrm{miR}-10$ and $\mathrm{miR}-317-3 p$ regulate the expression of four genes of interest. FTZ-F1 homologues, an essential factor regulating sex determination in mammals, have been identified in humans, mice, and a number of teleost species (Lala et al., 1992; Oba et al., 1996; Zhang et al., 2007; Von et al., 2011). FEM1 is a key signal-transducing regulator in the Caenorhabditis elegans sex-determination pathway, which influences the sex determination in $C$. elegans (Gaudet et al., 1996). High levels of forkhead box protein L2 (Fox/2), which plays a crucial role in the regulation of female sex-differentiation and development of the ovary, were also observed (Berta et al., 2012; Sandrine et al., 2012; Ashida et al., 2013). The extra sex combs (Esc) gene, first identified in Drosophila, is primarily expressed in the early embryos. In M. nipponense, MnEsc has been previously speculated to regulate the pre-metamorphic larval changes in morphology and organogenesis (Zhang et al, 2013s).

Slow-tonic S2 tropomyosin (Sst) and slow tropomyosin isoform (Sti) are two novel genes involved in the sex-determination mechanism or formation and maintenance of the normal structure of androgenic gland (Jin et al., 2014). The miRNA miR-184 and miR-184-3p were predicted to be involved in the regulation of Sst and Sti expression. Therefore, these two miRNA may be involved in the formation or maintenance of normal function of the androgenic gland in $M$. nipponense, which must be validated by further research.

In conclusion, we identified novel miRNA were identified in the androgenic gland of M. nipponense. Using the high-throughput Illumina Solexa system, we identified 1,077 miRNA from small RNA libraries, with reference to the de novo androgenic gland transcriptome of $M$. nipponense (obtained from RNA-Seq) and the sequences uploaded to the miRBase21 database. A total of $8,248,76,011$, and 78,307 target genes were predicted in the EST and SRA sequences provided in NCBI, and the androgenic gland transcriptome of $M$. nipponense, respectively. Some potential sex-related miRNA were identified using the predicted target genes. The results of our study provides new information regarding the miRNA expression in $M$. nipponense, which could be a basis for further genetic studies on decapod crustaceans.

\section{Conflicts of interest}

The authors declare no conflict of interest.

\section{ACKNOWLEDGMENTS}

Research supported by the Freshwater Fisheries Research Center, China Central Governmental Research Institutional Basic Special Research Project of the Public Welfare Fund (\#2015JBFM11), the National Science \& Technology Supporting Program of the 12th Five-year Plan of China (\#2012BAD26B04), the Science \& Technology Supporting Program of Jiangsu Province (Grant \#BE2012334), three aquatic projects of Jiangsu Province (\#D2015-16), and three aquatic projects of Jiangsu Province (\#D2013-6).

\section{REFERENCES}

Asgari S (2011). Role of MicroRNAs in insect host-microorganism interactions. Front. Physiol. 2: 48. Asgari S (2012). MicroRNA functions in insects. Insect. Biochem. Mol. 43: 388-397. 
Ashida H, Ueyama N, Kinoshita M and Kobayashi T (2013). Molecular identification and expression of FOXL2 and DMRT1 genes from willow minnow Gnathopogon caerulescens. Reprod. Biol. 13: 317-324.

Berta C, Oli SC, Adrien G, David L, et al. (2012). The transcription factor FOXL2: At the crossroads of ovarian physiology and pathology. Mol. Cell Endocrinol. 356: 55-64.

Bartel DP (2004). MicroRNAs: genomics, biogenesis, mechanism, and function. Cell 116: 281-297.

Bureau of Fishery, Ministry of Agriculture, P.R.C. (2009). Fisheries Economic Statistics. China Agricultural Press, Beijing.

Cai $Y$ and Shokita S (2006). Report on a collection of freshwater shrimps (Crustacea: Decapoda: Caridea) from the Philippines, with descriptions of four new species. Raffles. B. Zool. 54: 245-270.

Feng JB, Li JL and Cheng X (2008). Research progress on germplasm resource exploitation and protection of Macrobrachium nipponense. J. Shanghai Fish. Univ. 17: 371-376.

Gaudet J, Vanderlst I and Spence AM (1996). Post-transcriptional regulation of sex determination in Caenorhabditis elegans: widespread expression of the sex-determining gene fem-1 in both sexes. Mol. Biol. Cell 7: 1107-1121.

Jin S, Fu H, Zhou Q, Sun S, et al. (2013). Transcriptome analysis of androgenic gland for discovery of novel genes from the oriental river prawn, Macrobrachium nipponense, Using Illumina Hiseq 2000. PLoS One 8: e76840.

Jin SB, Jiang SF, Xiong YW, Qiao H, et al. (2014). Molecular cloning of two tropomyosin family genes and expression analysis during development in oriental river prawn, Macrobrachium nipponense. Gene 546: 390-397.

Johnson SM, Grosshans H, Shingara J, Byrom M, et al. (2005). RAS is regulated by the let-7 microRNA family. Cell 120: 635-647.

Lala DS, Rice DA and Parker KL (1992). Steroidogenic factor I, a key regulator of steroidogenic enzyme expression, is the mouse homolog of fushi tarazu-factor I. Mol. Endocrinol. 6: 1249-1258.

Lee RC, Feinbaum RL and Ambros V (1993). The C. elegans heterochronic gene lin-4 encodes small RNAs with antisense complementarity to lin-14. Cell 75: 843-854.

Li FJ, Bai HK, Zhang WY, Fu HT, et al. (2015a). Cloning of genomic sequences of three crustacean hyperglycemic hormone superfamily genes and elucidation of their roles of regulating insulin-like androgenic gland hormone gene. Gene 56: 68-75.

Li FJ, Bai HK, Xiong YW, Fu HT, et al. (2015b). Molecular characterization of insulin-like androgenic gland hormone-binding protein gene from the oriental river prawn Macrobrachium nipponense and investigation of its transcriptional relationship with the insulin-like androgenic gland hormone gene. Gen. Comp. Endocrinol. [Epub Ahead of Print].

Ma KY, Feng JB, Lin JY and Li JL (2011). The complete mitochondrial genome of Macrobrachium nipponense. Gene 487: 160-165.

Ma KY, Lin JY, Guo SZ, Chen Y, et al. (2013). Molecular characterization and expression analysis of an insulin-like gene from the androgenic gland of the oriental river prawn, Macrobrachium nipponense. Gen. Comp. Endocrinol. 185: 90-96.

Malecha SR, Nevin PA, Ha P, Barck LE, et al. (1992). Sex ratios and sex-determination in progeny from crosses of surgically sex reversed freshwater prawns, Macrobrachium rosenbergii. Aquaculture 105: 201-218.

Manor R, Aflalo ED, Segall C, Weil S, et al. (2004). Androgenic gland implantation promotes growth and inhibits vitellogenesis in Cherax quadricarinatus females held in individual compartments. Invertebr. Reprod. Dev. 45: 151-159.

Oba K, Yanase T, Nomura M, Morohashi K, et al. (1996). Structural characterization of human Ad4bp (SF-1) gene. Biochem. Biophys. Res. Commun. 226: 261-267.

O'Hara SP, Splinter PL, Gajdos GB, Trussoni CE, et al. (2010). NFkappaB p50-CCAAT/enhancer-binding protein beta (C/ EBPbeta)-mediated transcriptional repression of microRNA let-7i following microbial infection. J. Biol. Chem. 285: 216-225.

Pitipol M, Amornrat P, Wilaiwan C and Marti TT (2013). Computational identification of Penaeus monodon microRNA genes and their targets. Songklanakarin J. Sci. Technol. 35: 143-148.

Sagi A and Cohen D (1990). Growth, maturation and progeny of sex-reversed Macrobrachium rosenbetgii males. World Aquacult. 21: 87-90.

Sagi A, Ra'anan Z, Cohen D and Wax Y (1986). Production of Macrobrachium rosenbetgii in momosex population: yield characteristes under intensive monoculture conditions in cages. Aquaculture 51: 265-275.

Sagi A, Cohen D and Milner $Y$ (1990). Effect of androgenic gland ablation on morphotypic differentiation and sexual characteristics of male freshwater prawns, Macrobrachium rosenbergii. Gen. Comp. Endocrinol. 77: 15-22.

Sandrine C, Adrien G, David L, Anne-Laure T, et al. (2012). The transcription factor FOXL2: At the crossroads of ovarian physiology and pathology. Mol. Cell. Endocrinol. 356: 55-64.

Schulte LN, Eulalio A, Mollenkopf HJ, Reinhardt R, et al. (2011). Analysis of the host microRNA response to Salmonella uncovers the control of major cytokines by the let-7 family. EMBO J. 30: 1977-1989.

Song YN, Shi LL, Liu ZQ and Qiu GF (2014). Global analysis of the ovarian microRNA transcriptome: implication for miR-2 and miR-133 regulation of oocyte meiosis in the Chinese mitten crab, Eriocheir sinensis (Crustacea:Decapoda). BMC Genomics 15: 547.

Tan TT, Chen MS, Jennifer AH, Norliana K, et al. (2013). Deep parallel sequencing reveals conserved and novel miRNAs in gill and hepatopancreas of giant fresh water prawn. Fish Shellfish Immun. 35: 1061-1069. 
Tomer V and Sagi A (2012). The insulin-like androgenic gland hormone in crustaceans: From a single gene silencing to a wide array of sexual manipulation based biotechnologies. Biotechnol. Adv. 6: 1543-1550.

Tomer V, Rivka M, Eliahu DA, Simy W, et al. (2009). Temporal silencing of an androgenic gland-specific insulin-like gene affecting phenotypical gender differences and spermatogenesis. Endocrinology 150: 1278-1286.

Tomer V, Rivka M, Eliahu DA, Simy W, et al. (2011). Expression of an androgenic-gland-specific insulin-like peptide during the course of prawn sexual and morphotypic differentiation. ISRN Endocrinol. 2011: 1-11.

Von HJ, Jones I, Karlsson J and Olsson PE (2001). Developmental expression patterns of FTZ-F1 homologues in zebrafish (Danio rerio). Gen. Comp. Endocrinol. 121: 146-155.

Wightman B, Ha I and Ruvkun G (1993). Posttranscriptional regulation of the heterochronic gene lin-14 by lin-4 mediates temporal pattern formation in C. elegans. Cell 75: 855-862.

Xu P, Vernooy SY, Guo M and Hay BA (2003). The Drosophila microRNA Mir-14 suppresses cell death and is required for normal fat metabolism. Curr. Biol. 13: 790-795.

Zhang WM, Zhang YY, Zhang LH, Zhao HH, et al. (2007). The mRNA expression of P450 aromatase, gonadotropin betasubunits and FTZ-F1 in the orange-spotted grouper (Epinephelus coioides) during 17 alpha-methyltestosterone- induced precocious sex change. Mol. Reprod. Dev. 74: 665-673.

Zhang YP, Qiao H, Zhang WY, Sun SM, et al. (2013a). Molecular cloning and expression analysis of two sex-lethal homolog genes during development in the oriental river prawn, Macrobrachium nipponense. Genet. Mol. Res. 12: 4698-4711.

Zhang YP, Fu HT, Qiao H, Jin SB, et al. (2013b). Molecular cloning and expression analysis of transformer-2 gene during development in Macrobrachium nipponense (de Haan). J. World Aquac. Soc. 44: 338-349.

Zhang YP, Jiang SF, Xiong YW, Sun SM, et al. (2013c). Molecular Cloning and Expression Analysis of Extra Sex Combs Gene during Development in Macrobrachium nipponense. Turk. J. Fish. Aquat. Sc. 13: 331-340. 